\title{
Maternal hyperglycaemia affects cardiac development
}

Women with type 1 diabetes mellitus or type 2 diabetes mellitus are known to be at increased risk of having children with congenital heart defects characterized by heterotaxia, but the underlying mechanisms have been unclear. Now, a team from Japan suggest that hyperglycaemia disrupts heart morphogenesis, leading to congenital heart defects, and propose a mechanism to explain this link.

Chikara Meno and colleagues examined embryos from mice with streptozotocininduced diabetes mellitus and nondiabetic controls. Embryos from the diabetic mice developed a left-right reversed heart structure, which indicates that the left-right axis formation is disrupted in the embryos of diabetic mice. Expression of Pitx2, which encodes a protein that is involved in left-right axis formation, was suppressed in the left lateral mesoderm of embryos from diabetic mice, but was unaffected in the control embryos.
Furthermore, cultured embryos in a highglucose medium lacked expression of Nodal and had reduced Notch signalling, which are both involved in left-right axis formation in the heart.

"We found that high levels of glucose affect the initial stage of left-right axis formation, which eventually causes right isomerism and congenital heart defects," explains Meno. "Human cases of heterotaxia might be explained in part by this mechanism." As babies with heterotaxia born to women with diabetes mellitus can have left isomerism, Meno and colleagues are now interested in determining what mechanisms underlie this asymmetry.

\section{Claire Greenhill}

Original article Hachisuga, M. et al. Hyperglycemia impairs left-right axis formation and thereby disturbs heart morphogenesis in mouse embryos. Proc. Natl Acad. Sci. USA doi:10.1073/pnas.1504529112 